\title{
Rendimiento neuropsicológico en la adicción a la cocaína: una revisión crítica
}

\author{
Iñaki Lorea, Javier Fernández-Montalvo, Javier Tirapu-Ustárroz, Natalia Landa, José J. López-Goñi
}

Fundación Argibide (I. Lorea, J. Tirapu-Ustárroz). Departamento de Psicología y Pedagogía Universidad Pública de Navarra (I. Lorea, J. Fernández-Montalvo, N. Landa, J.J. López-Goñi). Servicio de Neuropsicología y Neuropsiquiatría; Clínica Ubarmin; Servicio Navarro de SaludOsasunbidea (J. Tirapu-Ustárroz) Centro de Salud Mental de San Juan; Servicio Navarro de Salud-Osasunbidea (N. Landa) Pamplona, Navarra, España.

Correspondencia: Dr. Iñaki Lorea. Fundación Argibide. Iturrama, 7, entreplanta. E-31007 Pamplona (Navarra)

E-mail:

i.lorea@fundacionargibide.org

Aceptado tras revisión externa:

15.06.10.

Cómo citar este artículo: Lorea I, Fernández-Montalvo J, Tirapu-Ustárroz J, Landa $\mathrm{N}$

López-Goñi JJ. Rendimiento neuropsicológico en la adicción a la cocaína: una revisión crítica. Rev Neurol 2010; 51: 412-26

Introducción. El uso crónico de cocaína se ha asociado con un amplio rango de déficits neuropsicológicos que afectan a la atención, la memoria, el aprendizaje o las funciones ejecutivas, aunque no se ha encontrado un perfil único de afectación asociado a la adicción a esta sustancia.

Objetivo. Revisar los principales estudios de rendimiento neurocognitivo en adictos a la cocaína, con especial atención al método de investigación empleado.

Desarrollo. Existe una gran diversidad metodológica en la investigación del déficit cognitivo asociado a la adicción a la cocaína. Estas diferencias dificultan la comparación de resultados y la obtención de un perfil claro de alteraciones neurocognitivas. Aun así, la mayoría de investigaciones encuentran déficit neuropsicológico, casi siempre de tamaño medio, asociado a la adicción a la cocaína. Este déficit afecta a la atención, memoria verbal y visual, y funciones ejecutivas (memoria de trabajo, flexibilidad mental, fluidez verbal, inhibición y toma de decisiones). Algunos estudios arrojan resultados con un perfil de ejecución cognitiva disociado.

Conclusiones. El uso crónico de cocaína se asocia con un peor rendimiento cognitivo. Por lo tanto, la valoración cognitiva de adictos a la cocaína puede mejorar la comprensión de la naturaleza de las dificultades que estos pacientes exhiben en su vida cotidiana y, además, puede servir de referencia para orientar su tratamiento. Es posible que sutiles alteraciones cognitivas conlleven importantes consecuencias en la conducta de estos pacientes. Sin embargo, la evaluación neuropsicológica no forma parte aún de los protocolos habituales de evaluación de este trastorno. Por lo tanto, se propone incluir una evaluación neuropsicológica en los protocolos de evaluación inicial de estos pacientes y adaptar el tratamiento al déficit encontrado.

Palabras clave. Adicción a la cocaína. Atención. Funciones ejecutivas. Memoria. Neuropsicología. Rehabilitación.

\section{Introducción}

El estudio neurocientífico de los trastornos adictivos está proporcionando un enorme caudal de conocimiento sobre los mecanismos neurobiológicos y neuropsicológicos implicados en el desarrollo de las conductas adictivas, así como sobre las alteraciones neurocognitivas asociadas a la adicción a drogas. La investigación en esta última área es de gran relevancia, entre otras razones, por el tipo de tratamiento que habitualmente se ofrece para abordar esta patología. Los programas de rehabilitación generalmente ofrecen intervenciones con un marcado carácter cognitivo y requieren del paciente habilidades cognitivas para comprender, recordar y aplicar lo tratado en la psicoterapia. Por ejemplo, las técnicas propias de la entrevista motivacional [1] y la prevención de recaídas [2], de amplia utilización en los programas de tratamiento de adicciones, demandan un estado cognitivo preservado para ser aprovechadas de manera adecuada [3].

Un área de estudio de interés creciente es la investigación sobre el tipo y la magnitud de las altera- ciones neuropsicológicas que presentan estos pacientes. En general, la hipótesis más repetida apunta que el abuso de cocaína se acompaña de algunos déficit cognitivos en funciones definidas con un criterio amplio, como la atención, el aprendizaje, la memoria o las funciones ejecutivas [4]. Sin embargo, no todos los resultados apoyan esta premisa, por lo que nos encontramos lejos aún de establecer consensos sobre el patrón de afectación de estos sujetos, así como sobre la magnitud de dicha afectación [5].

En el presente trabajo se examinan los estudios más recientes sobre el funcionamiento neuropsicológico de adictos a la cocaína. Como el interés primordial es comprobar los resultados del uso crónico de la sustancia, se revisan principalmente los estudios de pacientes abstinentes incluidos en protocolos de investigación o tratamiento.

\section{Cuestiones metodológicas}

Un aspecto que destaca en el análisis de los estudios sobre el déficit neurocognitivo en adictos a la 
cocaína es la heterogeneidad de los resultados obtenidos. Así por ejemplo, Horner [3] y Jovanovski et al [4], en sendas revisiones de estudios neuropsicológicos en adictos a cocaína, coinciden al señalar varios aspectos que diferencian de manera significativa el enfoque y método de las investigaciones y que pueden explicar la divergencia en sus resultados.

En primer lugar, los estudios neuropsicológicos no evalúan un sólo componente cognitivo, sino que la mayoría de los test empleados son sensibles a un conjunto de funciones neuropsicológicas integradas, como las perceptivas, motoras o ejecutivas. Por otra parte, la conceptualización de algunos procesos cognitivos, como la atención o las funciones ejecutivas, es demasiado general y se necesitan estudios de subprocesos más específicos. Además, algunos trabajos difieren al definir el tipo de función que mide cada instrumento: por ejemplo, mientras que Horner [3] se refiere al Trail Making Test (TMT) como un instrumento sensible a los déficit de atención sostenida o de velocidad de procesamiento de la información, Gillen et al [6] se refieren a él como una herramienta que mide integración visuomotora.

En segundo lugar, el tamaño de las muestras empleadas suele ser reducido (por lo general, no más de 40 adictos a la cocaína), ya que resulta difícil estudiar grandes muestras de adictos a la cocaína 'puros', que no tengan otra serie de trastornos que puedan afectar los resultados neuropsicológicos (por ejemplo, adicción al alcohol). Entre los estudios incluidos en esta revisión, sólo tres superan los 60 sujetos en el grupo clínico [7-9], mientras que la mayor parte no superan los 30 [6,10-12]. Esta circunstancia limita la generalización de los resultados.

En tercer lugar, la duración de la abstinencia a la cocaína es muy variable, y oscila entre varios días en unos estudios y varios meses en otros. Además, el período mínimo de abstinencia necesario para que los resultados cognitivos no se vean afectados por la sintomatología de abstinencia no se ha determinado con unanimidad. En esta revisión se han incluido tanto trabajos que requerían abstinencia a la cocaína 'en los días previos' a la evaluación neuropsicológica [13], como otros estudios en los que los sujetos presentaban una media de 40 días de abstinencia [14]. Asimismo, existen investigaciones que, dentro del mismo grupo clínico, incluyen sujetos con una gran variabilidad de tiempo de abstinencia, entre 13 y 1.466 días [5].

Tampoco se controla con igual rigor el cumplimiento de la abstinencia de los sujetos que participan en los estudios: algunos requieren la utilización de pruebas de consumo de drogas en orina o análisis de alcohol exhalado previos a las pruebas cogni- tivas [10,15], mientras que otros no controlan de manera objetiva el consumo de sustancias o no lo explicitan, con lo que la duración de la abstinencia informada resulta menos fiable $[6,11,16]$.

Además, los estudios difieren en cuanto a la gravedad, cantidad y duración del consumo de cocaína. Existen divergencias en el tipo de vía utilizada para la autoadministración de cocaína: la mayoría de estudios está realizada con consumidores de 'base libre', mientras que otros utilizan adictos que toman cocaína de forma esnifada o intravenosa. Igualmente, las estimaciones de gravedad se realizan utilizando criterios diferentes: mientras que unos estudios valoran el consumo de cocaína con la medida de los gramos por semana o mes de consumo $[6,16]$, otros valoran este indicador a través de medir el gasto de dinero al mes en cocaína $[10,13]$.

En sexto lugar, también existen diferencias en el consumo de otras sustancias además de la cocaína: la mayor parte de los estudios incluye sujetos que abusan de alcohol $[6,7,14,17,18]$, mientras que otros los excluyen $[10,12,15]$. Como consecuencia, la interpretación y comparación de los resultados es más difícil, ya que los déficit encontrados podrían atribuirse, por ejemplo, a la acción del abuso de alcohol más que al uso de cocaína.

Asimismo, se sabe que las características premórbidas de los sujetos estudiados pueden influir en su rendimiento cognitivo. Los traumatismos craneoencefálicos pasados, las enfermedades neurológicas, como las convulsiones, o digestivas, como la hepatitis, y los trastornos psiquiátricos comórbidos, como el déficit de atención con hiperactividad, pueden afectar por sí mismos a la ejecución en las pruebas neuropsicológicas. La falta de control sobre este tipo de variables puede confundir la interpretación de resultados de ejecución cognitiva [6].

También el tipo de grupo de control elegido para comparar los datos varía notablemente. Algunos estudios utilizan un grupo de control de características similares al grupo de adictos a la cocaína con rigurosos criterios de exclusión [11,15], mientras que otros no requieren de estos criterios $[5,7,19]$, o utilizan las muestras normativas que proporcionan los tests [20], disminuyendo así la comparación de los datos obtenidos.

Finalmente, otra circunstancia que dificulta la posibilidad de comparar los resultados es el empleo de diferentes pruebas para medir una misma función y el hecho de que un test requiera más de una función para su ejecución [4,21]. La falta de estudios que utilicen las mismas medidas de evaluación en diferentes muestras de pacientes imposibilita extraer conclusiones sólidas sobre la relación entre la 
naturaleza y magnitud del déficit cognitivo y de otras variables demográficas o clínicas. En conjunto, estas divergencias metodológicas pueden estar en la base de la diversidad de resultados que se han encontrado en los estudios neuropsicológicos realizados con adictos a cocaína.

\section{Estudios sobre rendimiento cognitivo en adictos a la cocaína}

Un esquema de las investigaciones principales se expone en la tabla.

\section{Estudios sobre la atención}

La evaluación de la atención es de particular importancia para el tratamiento de la adicción a sustancias, ya que los sujetos con déficit atencionales podrían beneficiarse menos de los tratamientos de deshabituación [3]. Desde un punto de vista conceptual, la atención agrupa diferentes subprocesos, como [22] la atención selectiva, la atención sostenida, la atención dividida y la velocidad de procesamiento de la información. Resulta difícil delimitar qué tipo de subcomponentes atencionales se hallan más afectados en los adictos a la cocaína [4]. Además, la atención subyace a otro tipo de funciones cognitivas, como la memoria o la resolución de problemas, habilidades de cuyo funcionamiento adecuado se requiere tanto en los tratamientos de rehabilitación como en las situaciones que se afrontan en la vida cotidiana $[23,24]$.

Varios trabajos han hallado déficit en pruebas que requieren algunos subcomponentes atencionales como la atención focalizada o selectiva, la vigilancia-atención sostenida, o la atención dividida. Los estudios de Rosselli et al [25], Goldstein et al [5], Cunha et al [11], Browndyke et al [26], Abi-Saab et al [7], Kübler et al [12], Pace-Schott et al [27], Ruiz et al [19] y Woicik et al [28] han coincidido al encontrar que los adictos a la cocaína presentan déficit atencionales, en comparación con sus respectivos grupos de control. Aunque, en general, el tamaño del efecto del déficit atencional encontrado se sitúa por encima de la media [4] y son numerosos los estudios que hallan estas funciones alteradas, también se encuentran algunos trabajos que no hallan diferencias significativas entre los adictos y el grupo de control $[13,29,30]$ o, incluso, otros que encuentran diferencias en algunos subcomponentes atencionales, como la atención focalizada, pero no en otros, como la atención sostenida [25]. Cuando se emplean paradigmas de investigación animal, el consumo prolongado ( $6 \mathrm{~h} /$ día), y no así el de menor intensidad ( $1 \mathrm{~h} /$ día), ha demostrado ser capaz de producir una afectación en la atención sostenida, lo que señala la capacidad de esta sustancia para provocar daño cognitivo drogoinducido [31].

Cabe destacar, por la especificidad de la metodología empleada y de los mecanismos atencionales analizados, el trabajo de Kübler et al [12], en el que estudiaron exclusivamente la atención alternante. En esta investigación, los adictos a la cocaína mostraron menos capacidad que los controles para cambiar el foco atencional, principalmente al trabajar con información de tipo visual, y esta dificultad se incrementaba en relación con la complejidad de las tareas realizadas. Un funcionamiento deficitario en esta habilidad podría afectar gravemente a la capacidad de los adictos de cambiar el foco atencional de un pensamiento a otro y favorecer así los pensamientos rumiativos que quedan fijados en aspectos relacionados con el uso de la cocaína durante el craving. De esta manera, los adictos con dificultades cognitivas en la atención alternante serían más proclives a la recaída, al presentar mayores problemas en el uso de estrategias cognitivas de distracción.

En resumen, aunque los estudios de funcionamiento cognitivo en adictos a la cocaína reflejan que las funciones atencionales se encuentran alteradas, en la actualidad no es posible delimitar un perfil concreto de deterioro atencional asociado a la adicción a la cocaína, y se necesitan estudios dirigidos específicamente a cada subcomponente atencional para determinar con exactitud qué aspectos de la atención pueden encontrarse más dañados [4]. Con toda probabilidad, la diversidad metodológica referida anteriormente está en la base de la dispersión de estos resultados. Además, la falta de consenso respecto a los modelos de funcionamiento de la atención y la consecuente evaluación de esta función según paradigmas diferentes dificulta la comparación de resultados.

\section{Estudios sobre la memoria}

Los estudios que han analizado el funcionamiento de la memoria en adictos a la cocaína concuerdan al señalar la presencia de un déficit de memoria, que afecta tanto a la memoria verbal $[5,13,20,25$, $27,28,30,32]$ como a la memoria visual $[5,11,12,19$, $20,25,33,34]$. Estos resultados sugieren que los pacientes adictos a la cocaína pueden tener problemas en el almacenamiento y recuperación de nueva información, tanto verbal como visual, posiblemente en relación con alteraciones funcionales de los lóbulos frontales y temporales [11]. En algunos casos, 
Tabla. Revisión de estudios sobre rendimiento neuropsicológico en adictos a la cocaína.

\begin{tabular}{|c|c|c|c|}
\hline Autor y año & $n$ & Pruebas empleadas & Principales resultados \\
\hline Ardila et al, 1991 & $36 \mathrm{AC}$ & $\begin{array}{l}\text { WMS, figura de Rey, fluencia verbal, Boston } \\
\text { Naming Test, WCST, dígitos del WISC }\end{array}$ & $\begin{array}{l}\text { Ejecución más baja que el grupo normativo en atención y memoria verbal } \\
\text { El déficit hallado es moderado } \\
\text { Correlación entre años de consumo de cocaína y déficit en atención: } \\
\text { apoyo a la hipótesis del déficit drogoinducido }\end{array}$ \\
\hline O'Malley et al, 1992 & $\begin{array}{l}20 \mathrm{AC} \\
20 \text { controles (GC) }\end{array}$ & $\begin{array}{l}\text { WAIS-R, BCT, Neuropsychological Screening } \\
\text { Battery, memoria verbal de Wechsler, figura } \\
\text { de Rey, Benton Multilingual Aphasia Exam, } \\
\text { Finger Oscillation Test }\end{array}$ & $\begin{array}{l}\text { Los AC superan a los controles en fluencia verbal } \\
\text { El 50\% de los AC presenta déficit frente a sólo el } 15 \% \text { del GC } \\
\text { Correlación entre cantidad y tiempo de abstinencia de cocaína y déficit } \\
\text { neuropsicológico: apoyo a la hipótesis del déficit drogoinducido }\end{array}$ \\
\hline Beatty et al, 1995 & $\begin{array}{l}24 \text { dependientes } \\
\text { de alcohol } \\
23 \mathrm{AC} \\
22 \mathrm{GC}\end{array}$ & $\begin{array}{l}\text { WMS, Face-Name Learning Test, WCST, } \\
\text { Conceptual Levels Analogy Test, aritmética } \\
\text { (WAIS-R), SILS Abstraction Subtest, TMT-A y B, } \\
\text { cubos y dígitos (WAIS), subtest de atención de } \\
\text { la Gordon Diagnostic Battery }\end{array}$ & $\begin{array}{l}\text { Alcohólicos y AC presentan déficit en: aprendizaje, memoria, abstracción, } \\
\text { velocidad psicomotora, perfil similar entre ambos } \\
\text { El déficit persiste al menos cinco semanas }\end{array}$ \\
\hline Hoff et al, 1996 & $\begin{array}{l}38 \mathrm{AC} \\
54 \mathrm{GC}\end{array}$ & $\begin{array}{l}\text { COWAT, WCST, BCT, BVRT, Stroop, TMT-A y B, } \\
\text { Finger Tapping, CT, BNT }\end{array}$ & $\begin{array}{l}\text { Los AC mejoran al GC en COWAT y número de categorías correctas del WCST } \\
\text { En el resto de pruebas, el déficit es leve (Booklet, TMT-B, Benton y Boston) }\end{array}$ \\
\hline Di Sclafani et al, 1998 & $\begin{array}{l}47 \mathrm{AC} \\
19 \text { controles }\end{array}$ & Batería MicroCog & $\begin{array}{l}\text { Los AC tienen peor rendimiento en pruebas de: índice global, abstracción, } \\
\text { habilidad espacial, memoria y aprendizaje } \\
\text { Correlación entre dosis máxima de cocaína y déficit neuropsicológico } \\
\text { No hay diferencias en la ejecución entre los AC y a cocaína + alcohol } \\
\text { Reserva funcional: el tamaño craneal está asociado a la ejecución cognitiva. Un } \\
\text { mayor número de neuronas hace al cerebro más resistente al daño por cocaína }\end{array}$ \\
\hline Gillen et al, 1998 & $\begin{array}{l}19 \text { varones } A C \\
16 \mathrm{GC}\end{array}$ & $\begin{array}{l}\text { BNT, CVLT, laberinto de Porteus, TMT-A y B, } \\
\text { JLO, WCST, COWAT, WAIS-R, memoria verbal } \\
\text { y visual de la WMS-III }\end{array}$ & $\begin{array}{l}\text { Los AC tienen peor rendimiento en tareas verbales y en tareas visuales } \\
\text { de secuenciación de estímulos } \\
\text { Los AC rinden mejor que GC en TMT-A }\end{array}$ \\
\hline Robinson et al, 1999 & $\begin{array}{l}30 \mathrm{AC} \\
30 \mathrm{AC}+\text { alcohol } \\
30 \mathrm{GC}\end{array}$ & $\begin{array}{l}\text { Halstead-Reitan Neuropsychological Test } \\
\text { Battery, WAIS-R, GP, Reitan-Klove Sensory } \\
\text { Perceptual Test, Aphasia Screening Test, Story } \\
\text { and Figure Memory Test }\end{array}$ & $\begin{array}{l}\text { El } 27 \% \text { de los AC presenta déficit, frente a sólo el 6,7\% en los AC + alcohol } \\
\text { y el 3,3\% en el GC } \\
\text { Déficit moderado en AC } \\
\text { Los AC tienen peores resultados que los AC + alcohol: apoyo a la hipótesis } \\
\text { de los efectos compensatorios entre cocaína y alcohol }\end{array}$ \\
\hline Bolla et al, 1999 & $\begin{array}{l}30 \mathrm{AC} \\
21 \mathrm{GC}\end{array}$ & $\begin{array}{l}\text { Controlled Oral Verbal Fluency, memoria } \\
\text { verbal de la WMS-III, RAVLT, figura de Rey, } \\
\text { Symbol Digit Test, CT, dígitos del WAIS-R, } \\
\text { TMT-A y B, WCST, figura de Rey, Stroop, JLO, } \\
\text { CCAP, Finger Tapping, GP }\end{array}$ & $\begin{array}{l}\text { La dosis de cocaína correlaciona inversamente con el rendimiento en función } \\
\text { ejecutiva, visuopercepción, velocidad psicomotora y destreza manual } \\
\text { Los AC son peores que el GC en tiempos de reacción, y mejores en memoria } \\
\text { reciente y habilidades visuoconstructivas } \\
\text { El déficit perdura por lo menos cuatro semanas }\end{array}$ \\
\hline Smelson et al, 1999 & $\begin{array}{l}35 \mathrm{AC} \\
17 \mathrm{GC}\end{array}$ & $\begin{array}{l}\text { Aritmética, dígitos, símbolos (WAIS-R), } \\
\text { Symbol Digit Modalities Test, GP, TMT-A y B }\end{array}$ & $\begin{array}{l}\text { Los AC son peores que el GC en cálculo mental, función motora y atención } \\
\text { alternante } \\
\text { El síndrome de abstinencia (cinco días de abstinencia media) afecta al flujo } \\
\text { sanguíneo cerebral, y éste a la ejecución cognitiva }\end{array}$ \\
\hline Van Gorp et al, 1999 & $\begin{array}{l}37 \mathrm{AC} \\
27 \mathrm{GC}\end{array}$ & CVLT, figura de Rey, Pursuit Rotor Task & $\begin{array}{l}\text { Los AC son peores que el GC en memoria declarativa y mejor en memoria } \\
\text { procedimental a los } 45 \text { días de abstinencia. }\end{array}$ \\
\hline Bolla et al, 2000 & $\begin{array}{l}29 \mathrm{AC} \\
27 \mathrm{AC}+\text { alcohol }\end{array}$ & $\begin{array}{l}\text { Vocabulario (WAIS-R), RAVLT, dígitos del } \\
\text { WAIS-R, TMT-A y B, Stroop, CCAP, GP }\end{array}$ & $\begin{array}{l}\text { Correlación entre dosis de alcohol y cocaína y un menor rendimiento cognitivo } \\
\text { La combinación de cocaína y alcohol afecta más al rendimiento cognitivo } \\
\text { que ambas por separado } \\
\text { Los efectos duran al menos } 4 \text { semanas } \\
\text { Reserva cognitiva: a los que hacen mejor la evaluación cognitiva al inicio } \\
\text { les influye menos la dosis de sustancias }\end{array}$ \\
\hline Serper et al, 2000 & $\begin{array}{l}21 \mathrm{AC} \\
34 \text { esquizofrénicos } \\
42 \mathrm{AC}+ \\
\text { esquizofrenia }\end{array}$ & CVLT & $\begin{array}{l}\text { Déficit similar en el grupo de cocaína y el grupo con esquizofrenia } \\
\text { Mayor déficit en el } A C+\text { esquizofrenia }\end{array}$ \\
\hline Roselli et al, 2001 & $\begin{array}{l}42 \mathrm{AC} \\
17 \mathrm{GC}\end{array}$ & $\begin{array}{l}\text { Dígitos y aritmética (WAIS-R), CVLT, figura de } \\
\text { Rey, WCST, BVRT, TMT-A y B, Stroop, fluencia } \\
\text { verbal, HVOT }\end{array}$ & $\begin{array}{l}\text { El déficit hallado es moderado } \\
\text { Correlación entre cantidad y duración del consumo de cocaína y el déficit } \\
\text { cognitivo: apoyo a la hipótesis del déficit drogoinducido }\end{array}$ \\
\hline Fillmore y Rush, 2002 & $\begin{array}{l}22 \mathrm{AC} \\
22 \mathrm{GC}\end{array}$ & Paradigma stop-signal & $\begin{array}{l}\text { Los AC muestran menos capacidad de inhibición que el GC: } \\
\text { necesitan más tiempo y cometen más errores por comisión }\end{array}$ \\
\hline
\end{tabular}


Tabla. Revisión de estudios sobre rendimiento neuropsicológico en adictos a la cocaína (cont.).

\begin{tabular}{|c|c|c|c|}
\hline Autor y año & $n$ & Pruebas empleadas & Principales resultados \\
\hline Bolla et al, 2004 & $\begin{array}{l}13 \mathrm{AC} \\
13 \mathrm{GC}\end{array}$ & Stroop modificado & $\begin{array}{l}\text { Metabolismo cerebral diferente entre grupos durante interferencia: en los AC, } \\
\text { menor activación del córtex cingulado anterior izquierdo y córtex prefrontal } \\
\text { dorsolateral derecho, y mayor activación del córtex cingulado anterior derecho } \\
\text { Dosis de cocaína relacionada con disminución de la activación del córtex } \\
\text { cingulado anterior y córtex prefrontal dorsolateral derecho } \\
\text { Los AC podrían utilizar diferentes circuitos neuronales que el GC para ejecutar } \\
\text { la tarea de interferencia: empleo de mecanismos compensatorios } \\
\text { El pequeño tamaño de la muestra puede influir para no hallar diferencias } \\
\text { en los resultados del Stroop }\end{array}$ \\
\hline Copersino et al, 2004 & $\begin{array}{l}20 \mathrm{AC} \\
23 \mathrm{AC}+ \\
\text { esquizofrenia } \\
19 \text { esquizofrénicos } \\
20 \mathrm{GC}\end{array}$ & Stroop modificado, CVLT & $\begin{array}{l}\text { Los AC emplean más tiempo que el resto en palabra-color con estímulos } \\
\text { asociados a la cocaína (efecto interferencia) } \\
\text { Los AC tienen más dificultades que el resto para ignorar los estímulos relacionados } \\
\text { con la cocaína }\end{array}$ \\
\hline
\end{tabular}

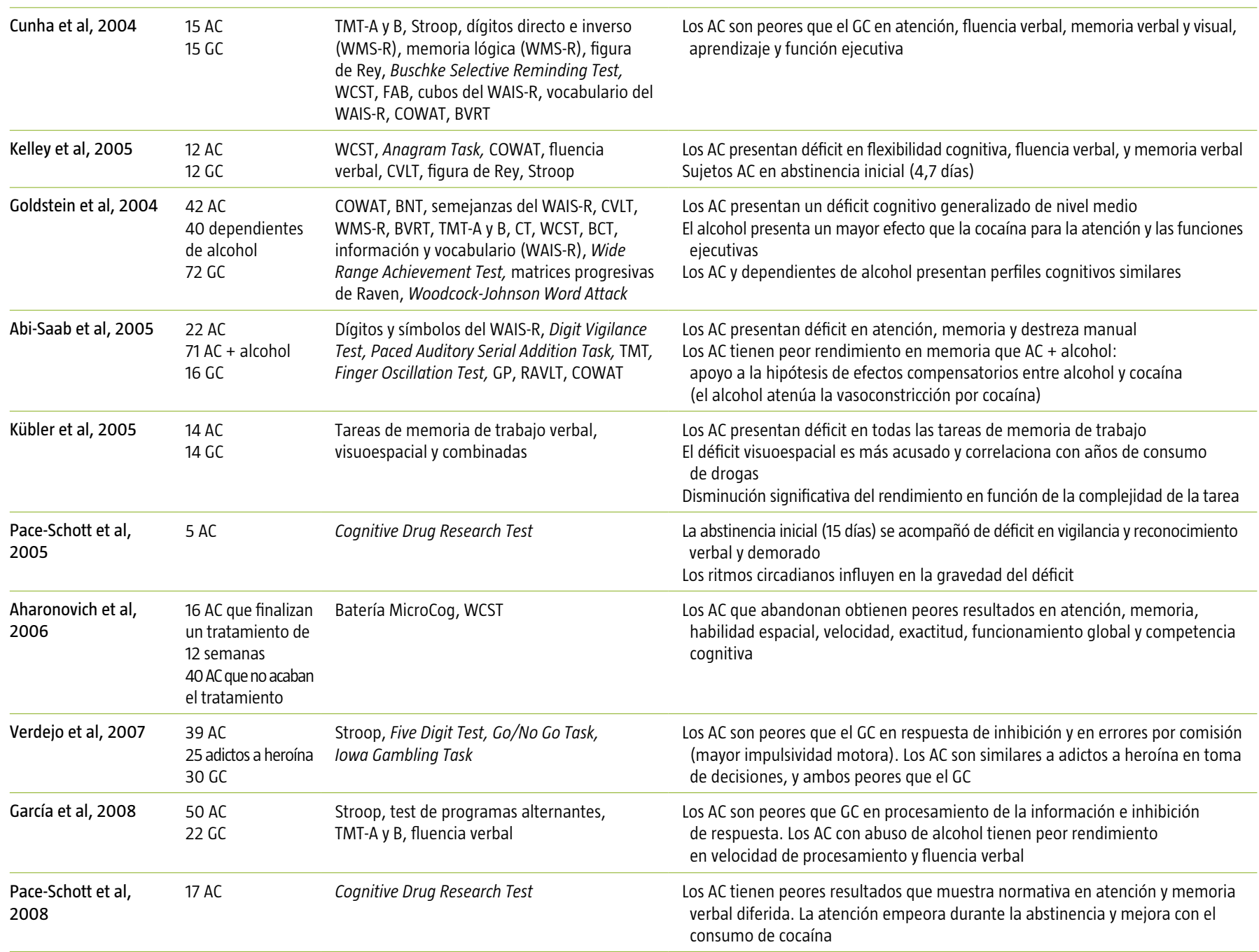


Tabla. Revisión de estudios sobre rendimiento neuropsicológico en adictos a la cocaína (cont.).

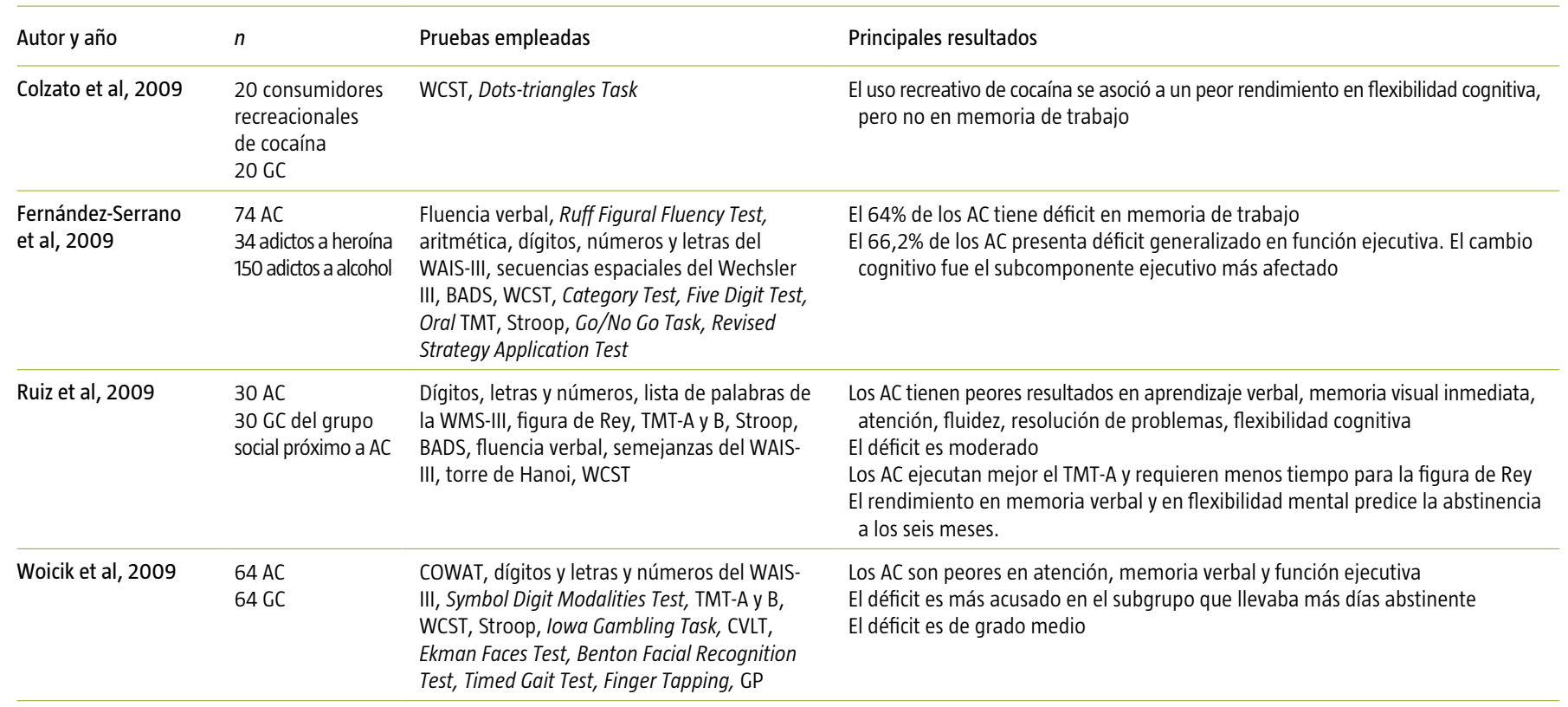

AC: adictos a la cocaína; BADS: Behavioural Assessment of the Dysexecutive Syndrome; BCT: Booklet Category Test; BNT: Boston Naming Test; BVRT: Benton Visual Retention Test; CCAP: California Computerized Assesment Package; COWAT: Controlled Oral Word Association Test; CT: Cancellation Test; CVLT: California Verbal Learning Test; FAB: Frontal Assessment Battery; GC: grupo control; GP: Grooved Pegboard; HVOT: Hooper Visual Organization Test; JLO: Judgment of Line Orientation; RAVLT: Rey Auditory Verbal Learning Test; SILS: Shipley Institute of Living Scale; TMT: Trail Making Test; WAIS: escala de inteligencia Wechsler para adultos; WCST: Wisconsin Card Sorting Test; WISC: escala de inteligencia Wechsler para niños; WMS: Wechsler Memory Scale.

los déficit de memoria presentan un considerable tamaño del efecto, asemejando los pacientes cocainómanos a pacientes esquizofrénicos [32].

Asimismo, se ha encontrado que el déficit en la memoria verbal es más acusado durante la abstinencia prolongada que durante la abstinencia inicial (72 horas) [28], hecho que puede ser consecuencia del efecto agudo de la cocaína sobre la cognición $[35,36]$. Este resultado puede ser relevante para el tratamiento, ya que puede menoscabar la capacidad para aprender los contenidos psicoeducativos que se incluyen en los programas de deshabituación (información sobre prevención de recaídas, por ejemplo) [30] y relacionarse con el conocido riesgo de estos pacientes de abandonar el tratamiento de manera prematura.

Un subsistema cognitivo muy relacionado con el funcionamiento de la memoria es la denominada memoria de trabajo. Se ha propuesto que la memoria de trabajo serviría de nexo de unión entre la atención y las funciones ejecutivas, y que su función principal sería mantener y manipular la información en línea. En estudios con adictos a la cocaína, se ha encontrado un rendimiento deficitario durante la abstinencia reciente y un mayor déficit en la agenda visuoespacial que en el bucle articulatorio [12]. Otros estudios también han hallado déficit en la memoria de trabajo [11,26,33,37], cuando se ha estudiado a sujetos adictos. Asimismo, se han observado diferencias en el patrón de activación cerebral durante una tarea de tipo $n$-back, en la que los adictos mostraron un peor rendimiento que los controles [38].

Sin embargo, el consumo de cocaína no parece asociarse a déficit en la memoria de trabajo cuando el consumo de esta sustancia se realiza de manera recreacional, sin criterios de abuso o dependencia [39]. Otros estudios tampoco han hallado déficit en esta función durante varias evaluaciones realizadas a los largo de 15 días de abstinencia [27], e incluso han hallado suaves mejoras en la ejecución de tareas en las que está implicada la agenda visuoespacial [40]. De cualquier manera, parece existir consenso en delimitar que el déficit hallado en esta función es de un tamaño del efecto medio y que la ejecución empeora conforme aumenta el requerimiento de la tarea $[4,37,38]$.

No obstante, este déficit puede afectar de manera crucial al aprovechamiento terapéutico de los pacientes con afectación cognitiva, puesto que la retención de instrucciones complejas, la selección 
de información relevante en sesiones individuales o grupales o la generalización de lo aprendido en la terapia a otros contextos como el familiar o laboral pueden verse disminuidas [41].

\section{Alteraciones de las funciones ejecutivas}

El consumo prolongado y la adicción a diversas drogas también se han asociado consistentemente al deterioro de otras funciones neuropsicológicas, entre las que destacan, por su crucial relevancia clínica, las funciones ejecutivas. De forma genérica, estas funciones describen procesos de control de la cognición y de regulación conductual, que operan a través de diferentes funciones cognitivas relacionadas entre sí [42]. Más en concreto, estudios estadísticos [43-45] han sintetizado que las funciones ejecutivas pueden organizarse en función de una serie de cinco componentes o factores ejecutivos [46]: la memoria de trabajo (comentado en el apartado sobre la memoria), la fluidez verbal, la capacidad de inhibición, la flexibilidad cognitiva y la toma de decisiones.

La fluidez verbal es la capacidad para producir lenguaje de manera continua y se relaciona con el funcionamiento ejecutivo a través de la organización del pensamiento y del empleo de estrategias de búsqueda de palabras. Éste es uno de los subcomponentes del funcionamiento ejecutivo en el que se ha hallado un menor tamaño del efecto [4] al comparar el rendimiento de adictos a la cocaína con sujetos control. Como ya hemos referido con anterioridad, incluso se ha encontrado que un grupo de adictos presentaba mejores resultados que sujetos control [16]. Los adictos a la cocaína también exhiben una mayor fluidez verbal al nombrar palabras relacionadas con el consumo de sustancias [47], lo cual sugiere un sesgo atencional, con un mayor grado de 'sintonización', hacia estímulos relacionados con el consumo de sustancias $[48,49]$. No obstante, una mayoría de estudios ha encontrado déficit en la fluidez verbal de estos pacientes $[6,11,19,26,30,50]$. Respecto a la localización neuroanatómica de las estructuras asociadas al déficit, se han obtenido resultados mixtos, que señalan tanto una amplia distribución corticosubcortical de hipoperfusión [26] como una hipoperfusión más específica, circunscrita a regiones frontotemporales [50]. La repercusión del déficit en la fluidez verbal puede ser significativa para estos pacientes, puesto que una disminución en las estrategias de búsqueda puede limitar, durante un tiempo indeterminado, su capacidad de buscar nuevas actividades o usos del tiempo durante la rehabilitación [8].

La capacidad para controlar la respuesta impulsiva es otro importante dominio en el estudio de las funciones ejecutivas en adictos a la cocaína. No debemos olvidar que una de las conductas nucleares del abuso de drogas en general es la desinhibición y la pérdida de control sobre la propia conducta. A su vez, esta capacidad puede ser descompuesta en varios factores, como la capacidad para retrasar la gratificación, la capacidad para inhibir una respuesta preponderante, la reflexión previa a una conducta y otras $[51,52]$.

Los estudios muestran que los adictos a la cocaína presentan un déficit en la capacidad de inhibición asociado a la adicción a la cocaína. Estos resultados se han obtenido utilizando el paradigma de Stroop [53-55], el paradigma de stop-signal [56], el paradigma de go/no go [57] y el paradigma de delaydiscounting [58].

Parece que el déficit se hace más observable cuando las tareas aumentan el requerimiento cognitivo de la memoria de trabajo [59]. Asimismo, se ha observado que la impulsividad también se incrementa si se cambian las condiciones en las que un adicto aprende una tarea [52], dato que refleja la dificultad de estos sujetos en inhibir respuestas que previamente han sido reforzadas.

Existe un considerable acuerdo al señalar que el córtex cingulado anterior y el córtex orbitofrontal parecen ser estructuras neuronales que subyacen al control de la respuesta impulsiva [54,57,60,61], y que una hipofunción de estas áreas conlleva un empeoramiento en la respuesta de inhibición.

Aunque la investigación básica ha proporcionado modelos animales que señalan algunos déficit en procesos inhibitorios atencionales y conductuales a consecuencia del consumo repetido de cocaína [60, 62], en la actualidad también se contempla que el déficit en el control de impulsos puede predisponer al inicio en el consumo de drogas o favorecer la transición del uso de drogas al abuso [48,63].

De cualquier manera, paradigmas específicos de investigación también han hallado que el consumo agudo de cocaína puede mejorar el rendimiento de la función inhibitoria en adictos a la cocaína durante el síndrome de abstinencia inicial [51]. Se ha mantenido la hipótesis de que la hipofunción dopaminérgica causada por la adicción a la cocaína podría ser compensada por dosis bajas de cocaína que restaurarían, al menos temporalmente, los niveles de dopamina alterados. Este efecto podría significar un factor de riesgo de recaída en sujetos que inician un programa de deshabituación.

Por otra parte, el déficit en la capacidad de inhibición también puede interferir en el adecuado desarrollo de un tratamiento de deshabituación, provocando una toma de decisiones irreflexiva que em- 
puje a su abandono, o a realizar respuestas impulsivas sobre el consumo de sustancias [52].

La flexibilidad cognitiva es la capacidad para cambiar un criterio de conducta cuando los condicionantes externos o internos de la actividad se modifican, lo cual permite evitar errores por perseveración. Algunos déficit en la flexibilidad cognitiva se observan en el desarrollo de una adicción cuando el sujeto continúa administrándose una sustancia a pesar de que esto conlleva graves consecuencias para su vida. Este subcomponente de las funciones ejecutivas se ha encontrado afectado en varios estudios $[8,19,20,30,39,55]$. Parece que la afectación es de una magnitud media-leve [4,37], y que los resultados son de nuevo dependientes de la dificultad de la tarea: a mayor dificultad de la tarea, peor rendimiento. En este sentido, se han encontrado resultados más inconsistentes con el Wisconsin Card Sorting Test (WCST), mientras que, al utilizar tareas con un mayor requerimiento ejecutivo, los resultados son más homogéneos al indicar déficit $[4,6]$. Estos déficit se han relacionado con la disfunción del córtex orbitofrontal, amígdala basolateral y estriado ventral [64], núcleos que conforman un circuito que está implicado en la regulación de la conducta en función de sus consecuencias. Asimismo, se ha mantenido la hipótesis de que el déficit en estas funciones podría ser consecuencia de los cambios neuronales a largo plazo producidos por el consumo de drogas [65]. En esta línea, se ha encontrado que primates expuestos a cocaína disminuyen su flexibilidad cognitiva, sin que la cocaína afecte a otras funciones en las que la inhibición no está implicada [60].

Las repercusiones para el tratamiento de estos resultados pueden ser muy importantes, puesto que una de las demandas más frecuentes e inmediatas que se realiza a estos pacientes es cambiar sus hábitos de vida, mientras que estos resultados indican que algunos sujetos tendrían limitada la capacidad para adaptar su conducta a nuevos contextos, al menos temporalmente [30]. Por el contrario, considerar esta circunstancia puede mejorar el ajuste de las demandas que se realiza a estos pacientes, disminuyendo así la probabilidad de un abandono prematuro.

La toma de decisiones es un subcomponente de la función ejecutiva que aglutina mecanismos de mayor complejidad que los anteriores. Se ha definido la toma de decisiones como la capacidad de seleccionar la conducta más adaptativa para el organismo en función de una serie de alternativas. Esta habilidad requiere, por lo tanto, la valoración de aspectos cognitivos de la situación, las consecuencias de cada opción (recompensa-castigo) y las emociones asociadas a cada opción [46]. Se ha propuesto que este proceso se halla alterado en sujetos que desarrollan una adicción, ya que su toma de decisiones prioriza las alternativas a corto plazo ligadas al consumo de la sustancia y bloquea el aprendizaje en función de las experiencias negativas que ocurren como consecuencia, a medio-largo plazo, de la administración de la sustancia (perjuicios económicos, relacionales, laborales, de salud...) [66,67]. El sustrato neuroanatómico de esta función se sitúa en el córtex prefrontal ventromedial, que desempeña una función crítica al integrar las señales emocionales con aspectos cognitivos de cada alternativa. Varios trabajos han hallado alteraciones de neuroimagen en estas regiones en adictos a la cocaína [68,69].

Para evaluar esta habilidad, la prueba más utilizada es la Iowa Gambling Task [70]. En adictos a la cocaína, varios trabajos han encontrado una ejecución deficiente en esta prueba [37,52]. Además, se ha hallado que estos sujetos son los que menos aprenden con repeticiones sucesivas, en comparación con adictos al cannabis y sujetos controles [71]: este fenómeno parece relacionarse con una hipersensibilidad de los adictos a la cocaína a las recompensas, hecho que dificulta el aprendizaje en función de las pérdidas, mientras que el déficit de los consumidores de cannabis se asociaría a sesgos atencionales, resultado del consumo de esta sustancia. También se ha encontrado que el tiempo de abstinencia parece mejorar la toma de decisiones en estos sujetos [72].

Sin embargo, el estudio de Woicik et al [28], que realiza una amplia evaluación de funciones cognitivas, entre las que se encuentra la toma de decisiones, halló unos resultados sin diferencias con el grupo de control en la Iowa Gambling Task, mientras que el resto de las pruebas de función ejecutiva (WCST y Attention Network Test) se hallaban afectadas. Nuevamente, la especificidad de la muestra empleada (adictos con consumo actual de la sustancia en este estudio frente a adictos en tratamiento y con más control ambiental) se sugiere como un factor que puede influir en la obtención de estos resultados discrepantes.

En resumen, los estudios de funcionamiento cognitivo que han evaluado algún componente ejecutivo tienden a encontrar déficit significativos en sujetos adictos a la cocaína. Estos déficit son más consistentes si la medida del funcionamiento ejecutivo es multidimensional $[5,11]$ o se emplean pruebas de complejidad, elevada como el test de categorías de Booklet [16]. Sin embargo, los resultados son más confusos con el empleo de una medida clásica de funcionamiento ejecutivo como el Wisconsin. Esta prueba arrojó resultados de déficit en el grupo de cocaína en 
el estudio de Rosselli et al [25], en el número de categorías completadas y en el número de errores cometidos, pero no en las respuestas perseverativas. Además, en el estudio de Ardila et al [20], los resultados de esta prueba reflejaban un deterioro mínimo, de menos de una desviación estándar por debajo del grupo normativo. En los estudios de Simon et al [33] $y$ de Cunha et al [11] no se encontraron diferencias en la ejecución del WCST entre el grupo de cocaína y el grupo de control. Finalmente, en el estudio de Hoff et al [16], los adictos a la cocaína obtuvieron mejores puntuaciones que el grupo de control en el número de categorías correctas de esta prueba. Este fenómeno se ha interpretado $[4,16,59]$ en relación con la dificultad y multidimensionalidad de la medida: es más probable que los adictos a la cocaína muestren déficit ejecutivos en pruebas más complejas y multidimensionales, mientras que su ejecución se observa relativamente conservada en pruebas con un menor nivel de demandas ejecutivas.

\section{Estudios con perfil cognítivo disociado}

Como se ha señalado con anterioridad, los estudios sobre el déficit cognitivo observado en los adictos a la cocaína indican que un amplio conjunto de funciones se encuentra alterado, aunque en la actualidad no existe consenso sobre el tipo, la dirección o la magnitud de estos déficit. Buena muestra de ello la ofrecen varios trabajos que señalan un perfil de resultados disociado, en el que el grupo clínico (adictos a la cocaína) obtiene peores resultados que el grupo de control en algunas pruebas, pero, sorprendentemente, mejora la ejecución en otras. Dado lo inesperado de los resultados, dichos estudios se revisan a continuación de una manera detallada.

En el estudio de Hoff et al [16] sobre el efecto de la cocaína (en forma de 'crack') en el rendimiento cognitivo, compararon 38 adictos a 'crack'-cocaína con 54 sujetos de un grupo de control en una serie de medidas con afinidad por el funcionamiento de regiones frontales y temporales (formación de conceptos, flexibilidad mental y memoria). Los resultados arrojaban un patrón de deterioro disociado, ya que el grupo de cocaína ejecutaba mejor que el de control algunas pruebas 'frontales' (número de categorías correctas del WCST y fluencia verbal, por ejemplo) y peor otras (test de categorías de Booklet y TMT-B). Respecto a las pruebas de memoria, el grupo de cocaína obtuvo puntuaciones más bajas que el de control sólo en memoria espacial. En general, ni el deterioro ni la mejora de resultados asociados al consumo de cocaína fue de gran magnitud. Para explicar estos resultados divergentes, los autores se refieren a resultados similares hallados en estudios con pacientes psicóticos en los que el uso de metilfenidato mejoraba la fluencia verbal y la ejecución del WCST [73,74]. La influencia del metilfenidato sobre la modulación de la transmisión dopaminérgica podría ser responsable de estos resultados y la amplitud de la variable 'días de abstinencia de cocaína podría estar provocando una influencia similar, en este estudio, al producir diversos patrones de metabolismo de glucosa en sistemas dopaminérgicos (ganglios basales, córtex orbitofrontal, córtex frontal izquierdo) que explicarían la variabilidad de cambios cognitivos observados en el grupo de cocaína. Además, sugieren que la dificultad de la tarea podría ser un factor de influencia sobre los resultados: a menor dificultad, más probabilidad de que no se encuentren diferencias entre ambos grupos. Esta hipótesis explicaría los mejores resultados en el WCST, una tarea de tipo ejecutivo de menor dificultad que el test de Booklet, en la que adictos a la cocaína exhiben un peor rendimiento que los controles [75]. De cualquier manera, los autores también subrayan la limitación que significa no haber podido emparejar las características del grupo de control respecto a variables sociodemográficas (raza, nivel educativo, nivel socioeconómico), así como la falta de analíticas de orina que confirmaran la abstinencia informada por los pacientes.

Por su parte, Gillen et al [6] compararon 19 varones adictos a cocaína con 16 controles equivalentes en medidas sociodemográficas. En comparación con el grupo de control, los adictos realizaron peor las pruebas de contenido verbal -vocabulario e información de la escala de inteligencia Wechsler para adultos revisada (WAIS-R)-. El grupo de cocaína también ejecutó peor que el de control las pruebas de secuenciación visual y de fluencia verbal, tareas que requieren del funcionamiento adecuado de los lóbulos frontal y temporal. Sin embargo, encontraron que los adictos realizaban mejor que los controles el TMT-A, una sencilla tarea de atención sostenida y velocidad de procesamiento de la información, dato que no encuentra precedente en la investigación previa con este instrumento. Las pruebas de memoria, atención y abstracción no arrojaron diferencias significativas. Los autores sugieren que la peculiaridad de estos resultados podría ser similar a los hallazgos de otras investigaciones sobre la influencia que las drogas estimulantes producen en tareas sencillas de tipo perceptivomotor [76], donde se observa que bajas dosis de anfetamina se asocian a mejoras en la ejecución de pruebas simples de ejecución psicomotora. 
Otro patrón de disociación en el rendimiento cognitivo de adictos a la cocaína en tratamiento se encontró en un estudio sobre los sistemas de memoria declarativa y procedimental [15]. Se comparó un grupo de 37 adictos a cocaína con 27 controles en cuatro momentos diferentes de evaluación (admisión a tratamiento, a los 10 días, 21 días y 45 días después). Los resultados sobre la memoria declarativa señalaron un peor rendimiento en el grupo de cocaína en la prueba de memoria visual (figura de Rey de memoria), y no se encontraron diferencias en la prueba de memoria verbal (California Verbal Learning Test), mientras que los resultados en la tarea de memoria procedimental (Pursuit Rotor Task) indicaron un rendimiento superior, que aumentaba en los ensayos posteriores, respecto al nivel de ejecución del grupo de control.

Los autores sugieren que la recuperación progresiva de los niveles de dopamina que ocurre durante la abstinencia de cocaína y su interacción con el aumento de sensitividad de receptores $D_{1}$ y $D_{2}$, que sucede como adaptación a la depleción dopaminérgica contingente al consumo de cocaína, podría estar en la base de estos resultados. En definitiva, la recuperación progresiva de los niveles de dopamina, junto con las alteraciones producidas por la adicción en los receptores dopaminérgicos, podría subyacer a estos resultados sorprendentes, ya que produciría una hiperfunción dopaminérgica, de duración limitada, que promovería un mejor rendimiento en tareas de funcionamiento psicomotor.

Otra diferencia que quizás pueda resultar relevante en este estudio, en comparación con otros, es la que concierne al tiempo de consumo de cocaína a lo largo de la vida del grupo clínico. En este trabajo, los sujetos del grupo de cocaína presentaban una media de sólo 0,71 años de consumo de cocaína a lo largo de la vida. Este dato, junto con el requerimiento de abstinencia de alcohol de al menos seis meses, podría influir en la falta de déficit encontrada en el rendimiento neuropsicológico de los adictos a la cocaína.

Otro estudio que obtuvo mejores resultados en algunas pruebas neuropsicológicas en el grupo de adictos fue el realizado por Bolla et al [10]. En esta investigación, compararon a 30 adictos a la base de cocaína con 21 controles equivalentes en variables sociodemográficas, en una amplia batería de pruebas que evaluaban lenguaje, memoria verbal, memoria visual, funciones ejecutivas, visuopercepciónvisuoconstrucción, velocidad psicomotora y destreza manual. Se realizó la evaluación neuropsicológica tras 29 días de abstinencia de la cocaína, en una unidad de ingreso hospitalario.
Respecto a los resultados por grupos, el de control realizó peor que el experimental algunas tareas relacionadas con la memoria verbal (Rey Auditory Verbal Learning Test) y las funciones ejecutivas (cubos del WAIS-R). Por el contrario, el grupo de cocaína realizó peor que el de control las tareas de tiempos de reacción. No se hallaron diferencias significativas en el resto de las pruebas. Esta variabilidad, de difícil explicación, podría estar influida, al menos en parte, tal y como proponen los autores, por la falta de vigilancia hacia el grupo de control, puesto que no se registraron las actividades que habían realizado la noche anterior a la evaluación. De esta manera, los autores sospechan que el grupo de control pudiera haber falseado la información sobre las bebidas alcohólicas ingeridas en la noche anterior a la prueba, o sobre el número de horas que durmieron esa misma noche. Respecto a los resultados del grupo clínico, el grupo con un consumo de cocaína más grave mostró una peor ejecución que el resto, dato que apoya la hipótesis del déficit cognitivo, subyacente a alteraciones cerebrales frontales, producido por el consumo prolongado de cocaína.

Recientemente, y en nuestro ámbito, el grupo de investigación del CAD-4 de Madrid [19] ha estudiado el rendimiento cognitivo en un grupo de 30 adictos a la cocaína que realizaban un tratamiento ambulatorio. En comparación con el grupo de control, extraído del entorno social cercano de los pacientes, el grupo de adictos obtuvo peores resultados en pruebas de aprendizaje verbal, memoria visual inmediata, atención, fluencia verbal, abstracción, resolución de problemas y flexibilidad cognitiva. Sin embargo, superó significativamente a los controles en pruebas de velocidad de ejecución visuomotora. Los autores sugieren, en la misma línea de algunas argumentaciones anteriores, que este resultado podría deberse a una mejora en la ejecución producida por las sustancias estimulantes, mejora que puede objetivarse con más probabilidad en tareas con escasa sobrecarga atencional.

A modo de conclusión de este apartado, los resultados indican, en general, un deterioro sutil de funciones cognitivas, como la memoria visual (memoria espacial del Benton, figura de Rey) o algunos componentes de la función ejecutiva (TMT-B, test de Booklet, inhibición conductual, fluencia verbal). Por otra parte, se observan resultados heterogéneos en cuanto al perfil de rendimiento cognitivo, con resultados que mejoran significativamente al grupo de control en tareas con un componente tanto psicomotor-manipulativo (Rotor Pursuit Task, TMT-A, cubos del WAIS) como verbal (fluencia verbal, memoria verbal inmediata). Algunas hipótesis explica- 
tivas de estos resultados inesperados parecen coincidir $[6,15,16]$ al sugerir que fenómenos de neuroadaptación contingentes a la abstinencia podrían provocar una hiperfunción de los sistemas dopaminérgicos implicados en la ejecución de tareas sencillas (generación espontánea de palabras, TMT-A, número de categorías correctas el Wisconsin) o en las que se requiere de rapidez psicomotora (TMT-A, fluencia verbal, cubos del WAIS) o con un marcado componente manipulativo (Rotor Pursuit Task, cubos del WAIS). Por otra parte, también se ha señalado que quizás algunas pruebas de funcionamiento ejecutivo, como el WCST, no sean lo suficientemente sensibles para detectar algunos déficit sutiles que, sin embargo, podrían afectar de manera significativa al funcionamiento cognitivo [23] y, en consecuencia, al manejo del craving o de las situaciones de alto riesgo de recaída [5]. Finalmente, no debemos olvidar que las drogas psicoestimulantes pueden mejorar, bajo ciertas condiciones, el funcionamiento cognitivo [36,77,78], y este hecho puede significar un factor de influencia en los trabajos que ofrecen un perfil de ejecución disociado.

\section{Conclusiones}

La neurociencia aporta un nuevo nivel de análisis para comprender la adicción a la cocaína. Sin embargo, los estudios sobre la ejecución en tareas neuropsicológicas de adictos a la cocaína arrojan algunos resultados inconsistentes. Esto es consecuencia de, cuando menos, la complejidad metodológica de estos estudios, que ven condicionados sus resultados por una multitud de variables que son difíciles de controlar y replicar en trabajos posteriores.

En síntesis, los aspectos metodológicos que dificultan la comparación entre estudios se refieren a:

- El empleo de diferentes instrumentos para evaluar una misma función.

- El hecho de que la mayoría de test neuropsicológicos sean sensibles a la puesta en marcha de funciones cognitivas integradas, no específicas.

- El empleo de muestras pequeñas, que limitan la generalización de resultados.

- La variabilidad en el tiempo de abstinencia de cocaína de los sujetos que participan en los estudios y el diferente control de la abstinencia de drogas.

- La diversidad en la gravedad del abuso de sustancias, en parámetros como la cantidad de cocaína consumida, la vía de administración, la duración del consumo o la presencia de abuso de otras sustancias.
- La diversidad de los sujetos respecto a sus características premórbidas, como la presencia de otros trastornos psiquiátricos, enfermedades neurológicas, digestivas, traumatismos craneales.

- El empleo de diferentes grupos de comparación, como muestras normativas, o grupos de control de otros adictos, o de sujetos sin adicciones.

- El empleo de técnicas estadísticas basadas en el estudio de las 'diferencias estadísticamente significativas' y no del 'tamaño del efecto' de estas diferencias.

Estas variables influyen en los resultados de los estudios y han de tenerse en cuenta a la hora de interpretar y comparar unas investigaciones con otras.

En esta línea, algunas investigaciones han arrojado resultados con un perfil de ejecución disociado, en el cual los adictos mejoran el rendimiento de los controles en algunas pruebas, pero no en otras. Para explicar estos sorprendentes resultados, se han propuesto una serie de hipótesis que hacen referencia, en primer lugar, al conocido efecto de las sustancias psicoestimulantes de mejorar la ejecución en algunas tareas cognitivas sencillas. Asimismo, se ha sugerido la influencia de mecanismos de neuroadaptación contingentes a la abstinencia de cocaína, que podrían provocar una hiperfunción transitoria de los sistemas dopaminérgicos asociados a tareas de tipo psicomotor. También se ha señalado que la falta de vigilancia de las actividades que el grupo de control realizaba en los días del estudio podría afectar a su ejecución cognitiva, modificando así el punto de comparación del grupo experimental. Finalmente, se ha subrayado la importancia del grado de dificultad de las tareas, puesto que resulta más probable encontrar resultados disociados cuando las pruebas son más sencillas.

No obstante, la mayoría de trabajos ha hallado déficit cognitivo en los adictos a la cocaína. Existe cierto consenso al delimitar que el tamaño del déficit cognitivo es de grado medio, menor que el que suele observarse en otro tipo de patologías, como la esquizofrenia o el daño cerebral adquirido. Sin embargo, esta alteración sutil puede tener importantes consecuencias en la conducta de adictos atendidos en programas de rehabilitación, e interferir en su evolución a lo largo del tratamiento. Los estudios sobre el funcionamiento atencional son un buen ejemplo de ello. Algunos trabajos han hallado déficit en la atención alterna y lo han relacionado con la dificultad en el manejo del craving en estos pacientes, lo cual podría utilizarse en los entornos clínicos como un marcador cognitivo de vulnerabilidad hacia la recaída, para discriminar pacientes con ma- 
yor y menor riesgo. No obstante, se necesitan más estudios específicos sobre cada subsistema atencional. Además, es necesario tener en cuenta que la atención subyace a la mayor parte de las funciones cognitivas, como la memoria o la solución de problemas, lo cual subraya la importancia de la evaluación de cada subcomponente atencional.

También se ha observado que los adictos a la cocaína presentan déficit de memoria verbal y visual, aunque dichos déficit no se presentan siempre asociados. Parece que el déficit mnésico podría presentarse más atenuado durante la abstinencia inicial y ser más acusado más allá de la abstinencia inmediata, lo cual plantea consecuencias importantes para los programas de tratamiento de estos pacientes, dado que trabajan con contenidos y tareas que requieren de un adecuado funcionamiento de la memoria. Estos hallazgos podrían estar relacionados con la frecuente dificultad que exhiben algunos adictos en el aprendizaje de estrategias de prevención de recaídas.

La adicción a la cocaína también se ha asociado a un menor rendimiento en pruebas de funciones ejecutivas. Estos resultados se encuentran de forma más consistente con pruebas de mayor dificultad y si la evaluación se realiza durante períodos que van más allá de la abstinencia inicial. Se ha encontrado déficit en pruebas de memoria de trabajo, flexibilidad mental, fluidez verbal, inhibición y toma de decisiones. Aunque el tamaño del efecto suele ser medio-bajo, las potenciales implicaciones para el tratamiento pueden ser muy significativas. Sujetos con alteraciones ejecutivas pueden ver afectada su capacidad para retener instrucciones complejas, así como para seleccionar la información relevante. También pueden encontrarse con más dificultades para modificar su conducta y adaptarla a nuevos contextos. Asimismo, pueden verse afectados por una toma de decisiones impulsiva y que priorice las consecuencias a corto plazo de su conducta.

Como conclusión, cabe realizar una serie de sugerencias sobre la idoneidad y las utilidades de la evaluación neuropsicológica en el ámbito de las adicciones. Para ello, adaptaremos las propuestas por Ersche y Sahakian [79] y Yucel y Lubman [80] en el ámbito de los trastornos adictivos. En primer lugar, hay que insistir en que, a pesar de que se ha constatado la relevancia del estado cognitivo de los adictos para su buena evolución a lo largo del tratamiento, la evaluación neuropsicológica de estos pacientes aún ocupa un lugar secundario, en el mejor de los casos, en los protocolos de evaluación inicial. Sin embargo, la valoración cognitiva de adictos a la cocaína puede mejorar la comprensión de la natu- raleza de las dificultades que estos pacientes exhiben en su vida cotidiana. Parece razonable deducir que esta mejor comprensión mejorará la alianza terapéutica y, por ende, la retención en el tratamiento, verdadero caballo de batalla de los programas de rehabilitación de adicciones. Asimismo, la evaluación cognitiva puede servir de referencia para orientar el tratamiento en casos en los que se detecte déficit neuropsicológico.

En este último caso, se ha propuesto el empleo de información multimodal, que combine el canal verbal con el visual (haciendo mapas visuales de un proceso de toma de decisiones, por ejemplo). Asimismo, en el caso de que el déficit afecte al aprendizaje verbal, sería más adecuado acentuar el entrenamiento en habilidades de afrontamiento, antes que un enfoque centrado en la comprensión de aspectos psicodinámicos o relacionales. Otra vía de investigación en este ámbito tiene que ver con el desarrollo de fármacos que mejoren el funcionamiento cognitivo de estos pacientes. Los estudios realizados con el modafinilo y la atomoxetina representan algunos ejemplos de este interesante ámbito de estudio.

Este tipo de conocimiento nos permitirá acercarnos a entender la adicción a la cocaína (y cualquier otro trastorno mental) en términos de los procesos cerebrales alterados, y enfocar el tratamiento rehabilitador de una manera más específica, partiendo de las funciones más conservadas y afectadas y ofreciendo a cada paciente las actividades terapéuticas más necesarias para su caso.

\section{Bibliografía}

1. Miller W, Rollnick S. Motivational interviewing: preparing people to change addictive behavior. New York: Guilford Press; 1991.

2. Marlatt G, Gordon J. Relapse prevention. Maintenance strategies in the treatment of addictive behaviors. New York: Guilford Press; 1985.

3. Horner MD. Attentional functioning in abstinent cocaine abusers. Drug Alcohol Depend 1999; 54: 19-33.

4. Jovanovski D, Erb S, Zakzanis KK. Neurocognitive deficits in cocaine users: a quantitative review of the evidence. J Clin Exp Neuropsychol 2005; 27: 189-204.

5. Goldstein RZ, Leskovjan AC, Hoff AL, Hitzemann R, Bashan F, Khalsa SS, et al. Severity of neuropsychological impairment in cocaine and alcohol addiction: association with metabolism in the prefrontal cortex. Neuropsychologia 2004; 42: 1447-58.

6. Gillen RW, Kranzler HR, Bauer LO, Burleson JA, Samarel D, Morrison DJ. Neuropsychologic findings in cocaine-dependent outpatients. Prog Neuropsychopharmacol Biol Psychiatry 1998; 22: 1061-76.

7. Abi-Saab D, Beauvais J, Mehm J, Brody M, Gottschalk C, Kosten TR. The effect of alcohol on the neuropsychological functioning of recently abstinent cocaine-dependent subjects. Am J Addict 2005; 14: 166-78.

8. Fernández-Serrano MJ, Pérez-García M, Perales JC, 
Verdejo-García A. Prevalence of executive dysfunction in cocaine, heroin and alcohol users enrolled in therapeutic communities. Eur J Pharmacol 2010; 626: 104-12.

9. Turner TH, LaRowe S, Horner MD, Herron J, Malcolm R. Measures of cognitive functioning as predictors of treatment outcome for cocaine dependence. J Subst Abuse Treat 2009; 37: 328-34.

10. Bolla KI, Rothman R, Cadet JL. Dose-related neurobehavioral effects of chronic cocaine use. J Neuropsychiatry Clin Neurosci 1999; 11: 361-9.

11. Cunha PJ, Nicastri S, Gomes LP, Moino RM, Peluso MA. Neuropsychological impairments in crack cocaine-dependent inpatients: preliminary findings. Rev Bras Psiquiatr 2004; 26 : 103-6.

12. Kübler A, Murphy K, Garavan H. Cocaine dependence and attention switching within and between verbal and visuospatial working memory. Eur J Neurosci 2005; 21: 1984-92.

13. Di Sclafani V, Truran DL, Bloomer C, Tolou-Shams M, Clark HW, Norman D, et al. Abstinent chronic crack-cocaine and crack-cocaine/alcohol abusers evidence normal hippocampal volumes on MRI despite persistent cognitive impairments. Addict Biol 1998; 3: 261-70.

14. Di Sclafani V, Tolou-Shams M, Price LJ, Fein G. Neuropsychological performance of individuals dependent on crack-cocaine, or crack-cocaine and alcohol, at 6 weeks and 6 months of abstinence. Drug Alcohol Depend 2002; 66: 161-71

15. Van Gorp WG, Wilkins JN, Hinkin CH, Moore LH, Hull J, Horner MD, et al. Declarative and procedural memory functioning in abstinent cocaine abusers. Arch Gen Psychiatry 1999; 56: 85-9.

16. Hoff AL, Riordan H, Morris L, Cestaro V, Wieneke M, Alpert R, et al. Effects of crack cocaine on neurocognitive function. Psychiatry Res 1996; 60: 167-76.

17. Robinson JE, Heaton RK, O'Malley SS. Neuropsychological functioning in cocaine abusers with and without alcohol dependence. J Int Neuropsychol Soc 1999; 5: 10-9.

18. Bolla K, Funderburk FR, Cadet JL. Differential effects of cocaine and cocaine plus alcohol on neurocognitive performance. Neurology 2000; 54: 2285-92.

19. Ruiz-Sánchez de León JM, Pedrero-Pérez E, Llanero-Luque M, Rojo-Mota G, Olivar-Arroyo A, Bouso-Saiz JC, et al Neuropsychological profile in cocaine addiction: issues about addict's social environment and predictive value of cognitive status in therapeutic outcomes. Adicciones 2009; 21: 119-32.

20. Ardila A, Rosselli M, Strumwasser S. Neuropsychological deficits in chronic cocaine abusers. Int J Neurosci 1991; 57: 73-9.

21. Landa N, Fernández-Montalvo J, Tirapu-Ustárroz J. Alteraciones neuropsicológicas en el alcoholismo: una revisión sobre la afectación de la memoria y las funciones ejecutivas. Adicciones 2004; 16: 41-51.

22. Van Zomeren AH, Brouwer WH. Theories and concepts of attention. In Brouwer WH, ed. Clinical neuropsychology of attention. New York: Oxford University Press; 1994. p. 7-38

23. Aharonovich E, Hasin DS, Brooks AC, Liu XH, Bisaga A, Nunes EV. Cognitive deficits predict low treatment retention in cocaine dependent patients. Drug Alcohol Depend 2006; 81: 313-22.

24. Lezak M, Howieson D, Loring D. Neuropsychological assessment. 4 ed. New York: Oxford University Press; 2004.

25. Rosselli M, Ardila A, Lubomski M, Murray S, King K. Personality profile and neuropsychological test performance in chronic cocaine-abusers. Int J Neurosci 2001; 110: 55-72.

26. Browndyke JN, Tucker KA, Woods SP, Beauvais J, Cohen RA, Gottschalk PCH, et al. Examining the effect of cerebral perfusion abnormality magnitude on cognitive performance in recently abstinent chronic cocaine abusers. J Neuroimaging 2004; 14: 162-9.

27. Pace-Schott EF, Morgan PT, Malison RT, Hart CL, Edgar C, Walker $\mathrm{M}$, et al. Cocaine users differ from normal on cognitive tasks which show poorer performance during drug abstinence. Am J Drug Alcohol Abuse 2008; 34: 109-21.
28. Woicik PA, Moeller SJ, Alia-Klein N, Maloney T, Lukasik $\mathrm{TM}$, Yeliosof $\mathrm{O}$, et al. The neuropsychology of cocaine addiction: recent cocaine use masks impairment. Neuropsychopharmacology 2009; 34: 1112-22.

29. Smelson DA, Roy A, Santana S, Engelhart C. Neuropsychological deficits in withdrawn cocaine-dependent males. Am J Drug Alcohol Abuse 1999; 25: 377-81.

30. Kelley BJ, Yeager KR, Pepper TH, Beversdorf DQ. Cognitive impairment in acute cocaine withdrawal. Cogn Behav Neurol 2005; $18: 108-12$

31. Briand L, Flagel S, García-Fuster M, Watson S, Akil H, Sarter M, et al. Persistent alterations in cognitive function and prefrontal dopamine D2 receptors following extended, but not limited, access to self-administered cocaine. Neuropsychopharmacology 2008; 33: 2969-80.

32. Serper MR, Bergman A, Copersino ML, Chou JCY, Richarme D, Cancro R. Learning and memory impairment in cocaine-dependent and comorbid schizophrenic patients. Psychiatry Res 2000; 93: 21-32.

33. Simon SL, Domier CP, Sim T, Richardson K, Rawson RA, Ling W. Cognitive performance of current methamphetamine and cocaine abusers. J Addict Dis 2002; 21: 61-74.

34. Beatty WW, Katzung VM, Moreland VJ, Nixon SJ. Neuropsychological performance of recently abstinent alcoholics and cocaine abusers. Drug Alcohol Depend 1995; 37: 247-53.

35. Johnson BA, Roache JD, Ait-Daoud N, Wallace CL, Wells LT, Wang Y, et al. Effects of isradipine on cocaine-induced changes in cognitive performance in recently abstinent cocaine-dependent individuals. Int J Neuropsychopharmacol 2005; 8: 549-56.

36. Higgins PG, Barrow GI, Tyrrell DAJ, Isaacs D, Gauci CL The efficacy of intranasal interferon-alpha-2a in respiratory syncytial virus-infection in volunteers. Antiviral Res 1990; 14: 3-10.

37. Van der Plas E, Crone E, Van den Wildenberg W, Tranel D, Bechara A. Executive control deficits in substance-dependent individuals: a comparison of alcohol, cocaine, and methamphetamine and of men and women. J Clin Exp Neuropsychol 2009; 31: 706-19.

38. Tomasi D, Goldstein RZ, Telang F, Maloney T, Alia-Klein N, Caparelli EC, et al. Widespread disruption in brain activation patterns to a working memory task during cocaine abstinence. Brain Res 2007; 1171: 83-92.

39. Colzato LS, Huizinga M, Hommel B. Recreational cocaine polydrug use impairs cognitive flexibility but not working memory. Psychopharmacology 2009; 227: 225-34.

40. Pace-Schott EF, Stickgold R, Muzur A, Wigren PE, Ward AS, Hart CL, et al. Cognitive performance by humans during a smoked cocaine binge-abstinence cycle. Am J Drug Alcohol Abuse 2005; 31: 571-91.

41. Verdejo-García A, Pérez-García M. Profile of executive deficits in cocaine and heroin polysubstance users: common and differential effects on separate executive components. Psychopharmacology 2007; 190: 517-30.

42. Tirapu-Ustárroz J, Muñoz-Céspedes JM, Pelegrín-Valero C. Funciones ejecutivas: necesidad de una integración conceptual. Rev Neurol 2002; 34: 673-85.

43. Miyake A, Friedman NP, Emerson MJ, Witzki AH, Howerter A, Wager TD. The unity and diversity of executive functions and their contributions to complex 'frontal lobe' tasks: a latent variable analysis. Cogn Psychol 2000; 41: 49-100.

44. Miyake A, Friedman NP, Rettinger DA, Shah P, Hegarty P. How are visuospatial working memory, executive functioning, and spatial abilities related? A latent-variable analysis. J Exp Psychol Gen 2001; 130: 621-40.

45. Fisk JE, Sharp CA. Age-related impairment in executive functioning: updating, inhibition, shifting, and access. J Clin Exp Neuropsychol 2004; 26: 874-90.

46. Tirapu-Ustárroz J, García-Molina A, Luna-Lario P, Roig-Rovira T, Pelegrín-Valero C. Modelos de funciones y control ejecutivo (I). Rev Neurol 2008; 46: 684-92.

47. Woicik P, Moeller S, Alia-Klein N, Maloney T, Lukasik T, Yeliosof $\mathrm{O}$, et al. The neuropsychology of cocaine addiction: 
recent cocaine use masks impairment. Neuropsychopharmacology 2009; 34: 112-22.

48. Garavan $\mathrm{H}$, Hester R. The role of cognitive control in cocaine dependence. Neuropsychol Rev 2007; 17: 337-45.

49. Beatty WW, Borrell GK. Forms of knowledge, cognitive impairment and drug abuse: a demonstration. Prog Neuropsychopharmacol Biol Psychiatry 2000; 24: 17-22.

50. Strickland TL, Mena I, Villanuevameyer J, Miller BL, Cummings J, Mehringer CM, et al. Cerebral perfusion and neuropsychological consequences of chronic cocaine use. J Neuropsychiatry Clin Neurosci 1993; 5: 419-27.

51. Garavan H, Kaufman JN, Hester R. Acute effects of cocaine on the neurobiology of cognitive control. Philos Trans R Soc Lond B Biol Sci 2008; 363: 3267-76.

52. Verdejo-García AJ, Perales JC, Pérez-García M. Cognitive impulsivity in cocaine and heroin polysubstance abusers. Addict Behav 2007; 32: 950-66.

53. Copersino M, Serper M, Vadhan N, Goldberg B, Richarme $\mathrm{D}$, Chou J, et al. Cocaine craving and attentional bias in cocaine-dependent schizophrenic patients. Psychiatry Res 2004; 128: 209-18.

54. Verdejo-García AJ, López-Torrecillas F, De Arcos FA, Pérez-García M. Differential effects of MDMA, cocaine, and cannabis use severity on distinctive components of the executive functions in polysubstance users: a multiple regression analysis. Addict Behav 2005; 30: 89-101.

55. García-Fernández G, García-Rodríguez O, Secades-Villa R, Álvarez-Carriles J, Sánchez-Hervás E. Rendimiento neuropsicológico de pacientes en tratamiento por adicción a la cocaína. Salud Drogas 2008; 8: 11-28.

56. Fillmore MT, Rush CR. Impaired inhibitory control of behavior in chronic cocaine users. Drug Alcohol Depend 2002; 66: 265-73.

57. Kaufman J, Ross T, Stein E, Garavan H. Cingulate hypoactivity in cocaine users during a go-no go task as revealed by event-related functional magnetic resonance imaging. J Neurosci 2003; 23: 7839-43.

58. Verdejo-García A, López-Torrecillas F, Giménez CO, Pérez-García M. Clinical implications and methodological challenges in the study of the neuropsychological correlates of cannabis, stimulant, and opioid abuse. Neuropsychol Rev 2004; 14: 1-41.

59. Hester R, Garavan H. Executive dysfunction in cocaine addiction: evidence for discordant frontal, cingulate, and cerebellar activity. J Neurosci 2004; 24: 11017-22.

60. Olausson P, Jentsch J, Krueger D, Tronson N, Nairn A, Taylor J. Orbitofrontal cortex and cognitive-motivational impairments in psychostimulant addiction: evidence from experiments in the non-human primate. Ann N Y Acad Sci 2007; 1121: 610-38.

61. Bolla K, Ernst M, Kiehl K, Mouratidis M, Eldreth D, Contoreggi $\mathrm{C}$, et al. Prefrontal cortical dysfunction in abstinent cocaine abusers. J Neuropsychiatry Clin Neurosci 2004; 16: 456-64.

62. Boutros NN, Uretsky NJ, Lui JJ, Millana RB. Effects of repeated cocaine administration on sensory inhibition in rats: preliminary data. Biol Psychiatry 1997; 41: 461-6.

63. Verdejo-García A, Lawrence AJ, Clark L. Impulsivity as a vulnerability marker for substance-use disorders: review of findings from high-risk research, problem gamblers and genetic association studies. Neurosci Biobehav Rev 2008; 32: 777-810.
64. Stalnaker TA, Takahashi Y, Roesch MR, Schoenbaum G. Neural substrates of cognitive inflexibility after chronic cocaine exposure. Neuropharmacology 2009; 56 (Suppl 1): S63-72.

65. Robinson TE, Kolb B. Structural plasticity associated with exposure to drugs of abuse. Neuropharmacology 2004; 47: 33-46.

66. Verdejo-García A, Pérez-García M, Bechara A. Emotion, decision-making and substance dependence: a somatic-marker model of addiction. Curr Neuropharmacol 2006; 4: 17-31.

67. Verdejo-García A, Bechara A. A somatic marker theory of addiction. Neuropharmacology 2009; 56: 48-62.

68. Bolla KI, Eldreth DA, London ED, Kiehl KA, Mouratidis M, Contoreggi $\mathrm{C}$, et al. Orbitofrontal cortex dysfunction in abstinent cocaine abusers performing a decision-making task. Neuroimage 2003; 19: 1085-94.

69. Tucker KA, Potenza MN, Beauvais JE, Browndyke JN, Gottschalk PC, Kosten TR. Perfusion abnormalities and decision making in cocaine dependence. Biol Psychiatry 2004; 56: $527-30$

70. Bechara A, Damasio AR, Damasio H, Anderson SW. Insensitivity to future consequences following damage to human prefrontal cortex. Cognition 1994; 50: 7-15.

71. Verdejo-García A, Benbrook A, Funderburk F, David P, Cadet JL, Bolla KI. The differential relationship between cocaine use and marijuana use on decision-making performance over repeat testing with the Iowa Gambling Task. Drug Alcohol Depend 2007; 90: 2-11.

72. Bartzokis G, Lu PH, Beckson M, Rapoport R, Grant S, Wiseman EJ, et al. Abstinence from cocaine reduces high-risk responses on a gambling task. Neuropsychopharmacology 2000; 22: 102-3.

73. Bilder RM, Lieberman JA, Kim YJ, Alvir JM, Reiter G. Methylphenidate and neuroleptic effects on oral word production in schizophrenia. Neuropsychiatry Neuropsychol Behav Neurol 1992; 5: 262-71.

74. Goldberg TE, Bigelow LB, Weinberger DR, Daniel DG, Kleinman JE. Cognitive and behavioral-effects of the coadministration of dextroamphetamine and haloperidol in schizophrenia. Am J Psychiatry 1991; 148: 78-84.

75. Omalley S, Adamse M, Heaton RK, Gawin FH. Neuropsychological impairment in chronic cocaine abusers. Am I Drug Alcohol Abuse 1992; 18: 131-44.

76. Klorman R, Bauer LO, Coons HW, Lewis JL, Peloquin LJ, Perlmutter RA, et al. Enhancing effects of methylphenidate on normal young-adults cognitive-processes. Psychopharmacol Bull 1984; 20: 3-9.

77. Stillman R, Jones RT, Moore D, Walker J, Welm S. Improved performance 4 hours after cocaine. Psychopharmacology 1993; 110: 415-20.

78. Grilly DM, Nocjar C. Cocaine and vigilance task-performance of rats -effects of delay of reinforcement. Pharmacol Biochem Behav 1990; 37: 643-8

79. Ersche KD, Sahakian BJ. The neuropsychology of amphetamine and opiate dependence: implications for treatment. Neuropsychol Rev 2007; 17: 317-36.

80. Yucel M, Lubman DI. Neurocognitive and neuroimaging evidence of behavioural dysregulation in human drug addiction: implications for diagnosis, treatment and prevention. Drug Alcohol Rev 2007; 26: 33-9.

\section{Neuropsychological performance in cocaine addiction: a critical review}

Introduction. Chronic use of cocaine has been associated with a wide range of neuropsychological deficits affecting attention, memory, learning and executive functions. However, it has not been found a unique profile of impairment associated with cocaine addiction.

Aim. To review the studies about neurocognitive performance in cocaine addicted patients, with special attention to the research method used. 
Development. A great diversity in the research methodology of cognitive deficits associated with cocaine addiction has been used. These differences make difficult to compare results and obtain a clear profile of neuropsychological deficits. Even though, most of studies find neuropsychological deficits (mainly of medium size) associated with cocaine addiction. These problems are usually related to attention, verbal and visual memory and executive functions (working memory, mental flexibility, verbal fluency, inhibition and decision making). Moreover, in some studies a dissociated profile of cognitive performance has been found.

Conclusions. Chronic use of cocaine is associated with worse cognitive performance. Therefore, the cognitive assessment of cocaine addicted patients can improve the understanding of the nature of the difficulties that these patients exhibit in everyday life and also can help to guide the treatment. Probably, the presence of subtle cognitive changes has important consequences on the behaviour of these patients. However, neuropsychological performance is not usually assessed in the assessment guidelines of this disorder. Therefore, it is proposed to include a neuropsychological assessment for initial assessment of cocaine addicted patients, as well as to tailor treatment to the found deficit.

Key words. Attention. Cocaine addiction. Executive functions. Memory. Neuropsychology. Rehabilitation. 